\title{
Identifying Gender From Images of Faces
}

\author{
J.M.D.Zakir Hussain, M.K.Mariam Bee
}

\begin{abstract}
The objective of this project is to identify the gender of a person by looking at his/her photograph. This is a case of supervised learning where the algorithm is first trained on a set of female and male faces, and then used to classify new data. We have not taken genders other than Male and Female into account. A preliminary algorithm is run to make sure that an image is that of a human before classification begins.
\end{abstract}

\section{I.INTRODUCTION}

Previous research has shown that our brain has specialized nerve cells responding to specific local features of a scene, such as lines, edges, angles or movement. Our visual cortex combines these scattered pieces of information into useful patterns. Automatic face recognition aims to extract these meaningful pieces of information and put them together into a useful representation in order to perform a classification/ identification task on them.

While we attempt to identify gender from facial features, we are often curious about what features of the face are most important in determining gender. A re localized features such as eyes, nose and ears more important or overall features such as head shape, hair line and face contour more important?

There are a plethora of successful and robust face recognition algorithms on the web. Instead of using the inbuilt tools that they provide, we start building various

We look at how these methods perform on our data, discuss the relative advantages and disadvantages of these methods and investigate the limitations on accuracy posed by the dataset itself. The mathematical equations governing these methods will not be discussed in this report.

\section{DATA SET AN D PROCESSING}

The data we have is a set of high resolution colour images of 396 female faces and 389 male faces obtained from the M UCT database. All images are frontal view of the face. The database provides diversity of lighting, age and ethnicity.

The images also have variations in:

\begin{tabular}{|l|l|l|}
\hline Gender & Training Set & Test set \\
\hline Male & 200 & 169 \\
\hline
\end{tabular}

Table 1: Dataset of faces

In this project, we define misclassification error as: Error $=$ No of images misclassified $/ \mathrm{N}$ o of images

Revised Manuscript Received on August 14, 2019.

J.M.D.Zakir Hussain, Saveetha School of Engineering (SIMATS), Chennai, Tamilnadu, India. (Email: Jmdakber@gmail.com)

M.K.Mariam Bee, Saveetha School of Engineering (SIMATS), Chennai, Tamilnadu, India. (Email: Maraiamresearch09@gmail.com) subject's head rotation and tilt subject's facial expression subject's face/ $\mathrm{H}$ air accessories position of the face in the image

However, this challenging database was chosen to make room for improvements in the algorithm.

This data has been used in four different ways on a single algorithm so that we can study how sen- sitive it is to the data quality. We run a python script to center all the images in our database - by centering the images the faces are aligned at the axis of symmetry of the face. Hence, we have a set of centered and uncentered images. We also use colored (RGB) and $\mathrm{B} / \mathrm{W}$ versions of the given images. Colour images have been compressed to 140x140 pixels and $\mathrm{B} / \mathrm{W}$ to $64 \times 48$ pixels. We now have four differentdatasets: Dataset1(centered, RGB),

Dataset2(centered,B/ W),

Dataset3(uncentered,RGB) and

Dataset4(uncentered, B/ W).

The dataset has been split into training set and test set as summarized in the following table:

\begin{tabular}{|l|l|l|}
\hline Gender & Test error & $\begin{array}{l}\text { Testing } \\
\text { error }\end{array}$ \\
\hline male & 0.03 & 0.8 \\
\hline female & 0.28 & 0.14 \\
\hline
\end{tabular}

Table 2: Eigenface Method on Dataset 4

\begin{tabular}{|l|l|l|}
\hline Gender & Testing error & Test eroor \\
\hline male & 0.6 & 0.14 \\
\hline female & 0.11 & 0.16 \\
\hline
\end{tabular}

Table 3: Eigenface Method on Dataset 3

On Dataset 4, the algorithm show s very good recognition for males but a very poor one for females. We conclude here that the algorithm is basically identifying almost every new face to be male, hence contributing to the large error for females. The figure below demonstrates this. When a male face is projected onto the male eigenspace, the resultant

reduced- dimension vector matches the other male faces very well. But when a female face is projected onto the female eigenspace, the resultant reduced-dimension vector does not match the female faces very well. In- fact, it favor's females over males only about $28 \%$ of the time.

One disadvantage of PCA is that it cannot give you an intuitive sense of why the algorithm is favouring males. But upon looking at the data $w$ here the algorithm misclassifies the person, we conclude that female(red) and male(blue) Eigenfaces

Published By:

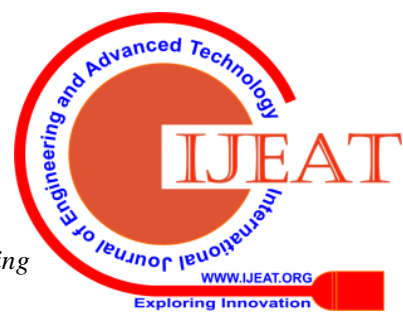




\section{IDENTIFYING GENDER FROM IMAGES OF FACES}

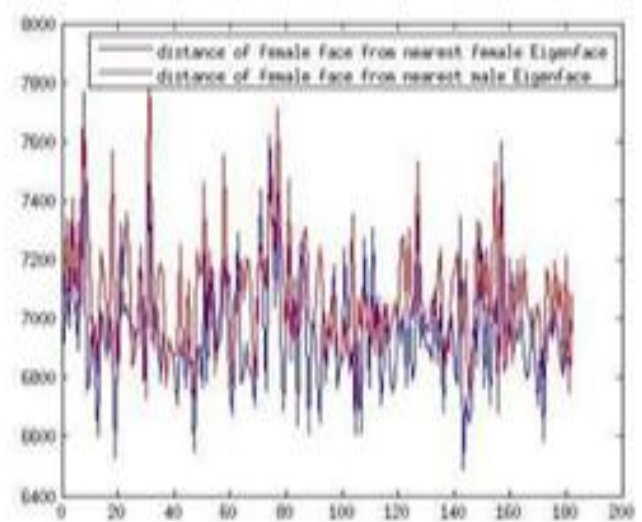

Figure 1: Plot of nearest distance of female faces in test set from female(red) and male(blue) Eigenfaces

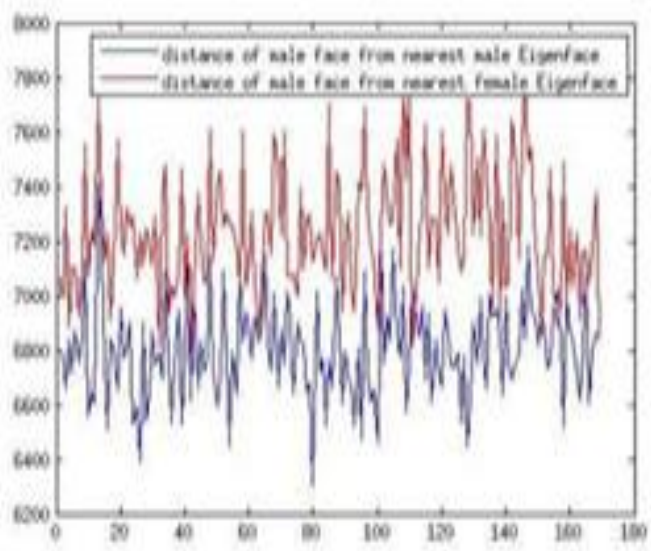

Running the same algorithm on Dataset 3 reduced the excessive bias towards males, as now the female faces were equally well-centered.

In all cases, the number of principal components was chosen to be 200 . We obtained this result by eliminating all eigen values whose value is zero. A $\mathrm{k}$-fold cross validation was performed to decide the number of dimensions in the reduced space more precisely. This resulted in a reduced dimension of 170

Below is a figure showing some images in the training set and the corresponding Eigenfaces:

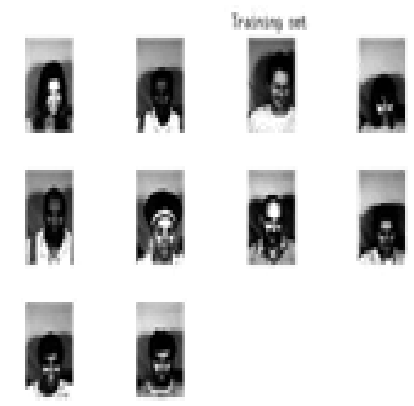

figure 3: A sample of training set data
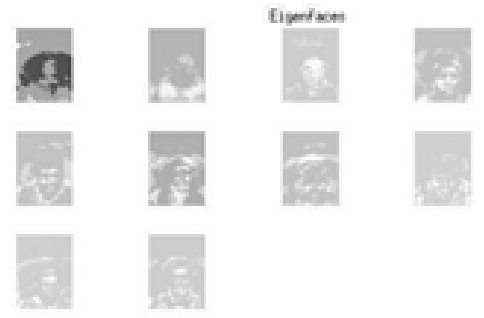

Figure 4: Eigenfaces of the above sample

K-mean $s$

We apply K-means directly on the pixel data that we get from images to obtain 10 clusters for female faces and 10 for male faces. We would like to call these the 10 most representative female and male faces. We then run the $\mathrm{K}$ Nearest Neighbors algorithm to classify our test images. K was chosen to be 5 after analyzing the performance of the algorithm (using cross validation) for all possible values of $\mathrm{K}$.

This is done on Dataset 3 and Dataset 4 . We get the following results:

\begin{tabular}{|l|l|}
\hline Gender & Test error \\
\hline male & 0.22 \\
\hline female & 0.16 \\
\hline
\end{tabular}

Table 4: K-means on Dataset 4

\begin{tabular}{|l|l|}
\hline Gender & Test error \\
\hline male & 0.12 \\
\hline female & 0.13 \\
\hline
\end{tabular}

Table 5: K-means on Dataset 3

\begin{tabular}{|l|l|}
\hline Gender & Test error \\
\hline male & 0.11 \\
\hline female & 0.11 \\
\hline
\end{tabular}

Table 6: PCA and GDA method on Dataset 2

\begin{tabular}{|l|l|}
\hline Gender & Test error \\
\hline male & 0.55 \\
\hline female & 0.7 \\
\hline
\end{tabular}

Table 7: PCA and GDA on Dataset 1

$\mathrm{k}$-fold cross validation was done to determine the number of PCA s required, and we found the optimal value to be 100. In order to visualize how GDA works with this data, we take 3 Principal Components and obtain the follow ing plot:

The Eigenface method classifies new data based on what the nearest vector is in terms of Euclidean dis- tance. Instead of using the nearest neighbour approach, we can perform 
supervised learning over the reduced space. GDA is one such attempt.

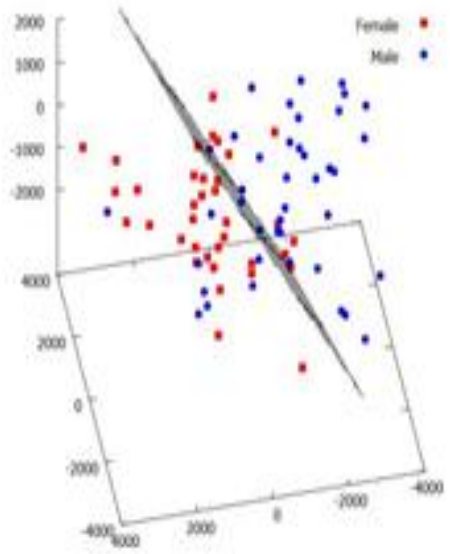

Figure 5: Implementing GDA for $\mathrm{K}=3$

\section{PCA WITH GDA}

The Eigenface method classifies new data based on what the nearest vector is in terms of euclidean dis- tance. Instead of using the nearest neighbour approach, we can perform supervised learning over the reduced space. GDA is one such attempt

\section{PCA WITH SVM}

SVM is yet another way of performing supervised learning over the reduced space.

$\mathrm{k}$-fold cross- validation was performed to chose the number of PCA s and 150 was found to be optimum. Cross validation was done for $\mathrm{k}=(10,20,30 . .200)$. This interval was arrived at after random sampling of k's. The PCA was applied to reduce dimensionality of the vectors that serve as inputs to the SVM.The SVM then does supervised learning. Sometimes this method is called the fisher discriminant analysis. Visualizing this data in the large dimensional space is hard, so we do it in 2D.We clearly need more attributes to classifythedata.

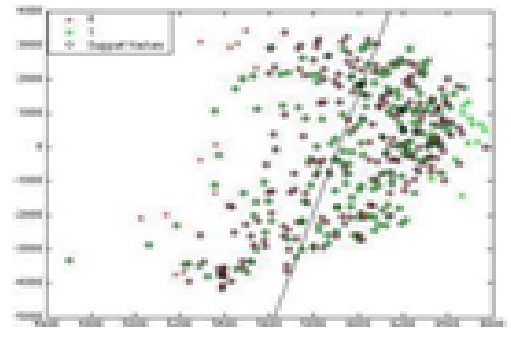

Figure 6: Implementing SVM for $\mathrm{K}=2$

Performance of this algorithm is :

\begin{tabular}{|l|l|}
\hline Gender & Test error \\
\hline male & 0.10 \\
\hline female & 0.13 \\
\hline
\end{tabular}

Table 8: PCA and SVM on Dataset 4

\begin{tabular}{|l|l|}
\hline Gender & Test error \\
\hline male & 0.90 \\
\hline female & 0.10 \\
\hline
\end{tabular}

Table 9: PCA and SVM on Dataset 3

\section{FISCHER FACES}

When PCA reduces the dimension in which we work, it definitely obtains the most representative reduced space. But it does nothing to make sure that these at- tributes also represent the salient differences between the male class and female class. Our algorithm's main aim should be to identify these features and give them highest priority while classifying them.

Fisher faces instead tries to maximize the variance between classes, instead of variance within a class. Hence it is much better suited for the gender classification ion task.
A $\mathrm{s}$ expected, Fisher Faces gives us remarkable re- sults of $10 \%$ on uncentered data and $3 \%$ on centered data. Also $10 \%$ is what all the algorithms converge to when used on uncentered data. This throw $\mathrm{s}$ light on the importance of centering it, as information about features can be very crucial in classifying it correctly.

\begin{tabular}{|l|l|}
\hline Gender & Test error \\
\hline male & 0.90 \\
\hline female & 0.11 \\
\hline
\end{tabular}

Table 10: Fisherface Method on Dataset 4

\begin{tabular}{|l|l|}
\hline Gender & Test error \\
\hline male & 0.25 \\
\hline female & 0.45 \\
\hline
\end{tabular}

Table 11: Fishface Method on Dataset 3

\section{HISTOGRAM OF ORIENTED GRADIENTS AND SVM \& RESULTS}

A s a foray into applying advanced and effective gender classification algorithms, we have used supervise SVM learning after extracting $\mathrm{H}$ OG descriptors of human faces. For this particular algorithm, we used code that was available online.

We carry out the scheme in B/ W space and use L-2 normalization for block normalization. For this method, images were not normalized during pre- processing. Also, the images were not centered because this method is invariant to geometric transformations of images.

A plot of gradients show what the most descriptive cues are that the SVM learns over. This is the only algo- rithm that can give us an insight as to w hich physical part of the face contributes most to gender detection.

The accuracy that this algorithm provides is the best of all. The algorithm also does not seem to be limited by the challenges that the data poses, giving us equally good results

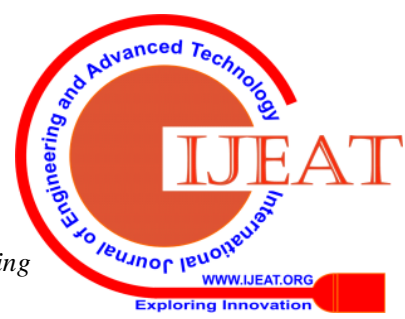




\section{IDENTIFYING GENDER FROM IMAGES OF FACES}

for both centered and uncentered data.

\begin{tabular}{|l|l|}
\hline Gender & Test error \\
\hline male & 0.17 \\
\hline female & 0.20 \\
\hline
\end{tabular}

Table 12: Fischer face Method on Dataset 3

\begin{tabular}{|l|l|}
\hline Gender & Test error \\
\hline male & 0.20 \\
\hline female & 0.23 \\
\hline
\end{tabular}

Table 13: Fischer face Method on Dataset 4

The gradient images of our dataset tells us that these are the fundamental differences between male and female faces:

The interior of a female face has softer face contoursFemale features are spread over larger areas than male features

The outline of a male face face is more rugged compared to a female face

Turns out that these differences are key in classi- flying a person to be male or female.

\section{DISCUSSION}

Gender classification algorithms can be of two types:

Pictorial: The algorithm reads pixel data into an array and uses statistical tools to process that array and make classification. Such algorithms require a dataset $\mathrm{w}$ here all images are properly aligned, without any noise.

Geometric: The algorithm reads pixel data and gets information on features such as width of jaw, curvature of cheek etc. It using this new information as the attribute space and provides an input to a supervised learning algorithm. These algorithms are more robust to geometric variations in dataset.

\section{FUTURE WORK}

Now that we have quantitative yardsticks for masculinity and feminists of a person, we could extend this know ledge to quantify what is perceived as beauty. Conventionally, beautiful people are known for epitomizing either masculinity or feminity. We use this hypothesis in devising our algorithm.

For this we need a dataset of images where each image is ranked on the basis of its at-great point edges.

\section{REFERENCES}

1. Turk M, Pentland A. Eigenfaces for recognition. Journal of Cognitive Neuroscience, 1991, 3(1): 71-86.

2. Freeman W, Adelson E. The design and use of steerable filters. IEEE Trans. Pattern Analysis and Machine Intelligence, 1991, 13(9): 891-906.

3. Belongie S, Malik J, Puzicha J. Shape matching and object recognition using shape contexts. IEEE Trans. Pattern Analysis and Machine Intelligence, 2002, 24(4): 509-522.

4. Zhao W, Chellappa R, Rosenfeld A, et al. Face recognition: A literature survey. ACM Comput. Surv., 2003, 35: 399-458.

5. Belhumeur P N, Hespanha J P, Kriegman D J. Eigenfaces vs. fisherfaces: Recognition using class specific linear projection. IEEE Trans. Pattern Analysis and Machine Intelligence, 1997, 19(7): 711-720.

6. Ahonen T, Hadid A, Pietikainen M. Face recognition with local binary pattern. In: Proc. 8th Eur. Conf. Computer Vision. Prague, Czech, 2004: 469-481.

7. Gabor D. Theory of communication. Journal of Institute for Electrical Engineering, 1946, 93(III): 429-457.

8. Lades M, Vorbruggen J C, Buhmann J, et al. Distortion invariant object recognition in the dynamic link architecture. IEEE Trans. Computers, 1993, 42(3): 300311.

9. Wiskott L, Fellous J M, Kruger N, et al. Face recognition by elastic bunch graph matching. IEEE Trans. Pattern Analysis and Machine Intelligence, 1997, 19(7): 775779 .

10. Freeman W T, Roth M. Orientation histograms for hand gesture recognition. In: Intl. Workshop on Automatic Face and Gesture Recognition. IEEE Computer Society, Zurich, Switzerland, 1995: 296-301.

11. Dalal N, Triggs B. Histograms of oriented gradients for human detection. In: IEEE Conference on Computer Vision and Pattern Recognition (CVPR). San Diego, CA, USA, 2005, 1: 886-893.

12. Lowe D G. Object recognition from local scale-invariant features. In: Proceedings of the 7th International Conference on Computer Vision. Kerkyra, Greece, 1999, 2: 1150-1157.

13. Granlund $\mathrm{G} \mathrm{H}$. In search of a general picture processing 224 Tsinghua Science and Technology, April 2011, 16(2): 216-224 operator. Computer Graphics and Image Processing, 1978, 8(2): 155-173. 